\title{
SELF-APPROXIMATION FOR THE RIEMANN ZETA FUNCTION
}

\author{
TAKASHI NAKAMURA and LUKASZ PAŃKOWSKI ${ }^{凶}$
}

(Received 10 May 2012; accepted 18 August 2012; first published online 31 October 2012)

\begin{abstract}
In the paper we deal with self-approximation of the Riemann zeta function in the half plane $\operatorname{Re} s>1$ and in the right half of the critical strip. We also prove some results concerning joint universality and joint value approximation of functions $\zeta(s+\lambda+i d \tau)$ and $\zeta(s+i \tau)$.
\end{abstract}

2010 Mathematics subject classification: primary 11K60; secondary 11M99.

Keywords and phrases: hybrid universality, the Riemann zeta function, self-approximation.

\section{Introduction}

Bohr in [3] proved that every Dirichlet series is almost periodic in its half plane of absolute convergence. More precisely, he showed that for any Dirichlet series $\sum_{n=1}^{\infty} a_{n} / n^{s}$ and for an arbitrary $s$ lying in the half plane of absolute convergence,

$$
\forall_{\varepsilon>0} \liminf _{T \rightarrow \infty} \frac{1}{T} \mu\left\{\tau \in[0, T]:\left|\sum_{n=1}^{\infty} \frac{a_{n}}{n^{s+i \tau}}-\sum_{n=1}^{\infty} \frac{a_{n}}{n^{s}}\right|<\varepsilon\right\}>0
$$

here $\mu\{A\}$ stands for the Lebesgue measure of a measurable set $A$.

Obviously, the above inequality holds for the Riemann zeta function in the half plane $\mathcal{D}:=\{s \in \mathbb{C}: \operatorname{Re}(s)>1\}$.

In 1981 Bagchi [1] discovered that the above property holds uniformly on compacta in the critical strip if and only if the Riemann hypothesis is true. For convenience, let $D:=\{s \in \mathbb{C}: 1 / 2<\operatorname{Re}(s)<1\}$ and $H(K)$ denote the space of nonvanishing continuous functions on a compact set $K$, which are analytic in the interior, equipped with the supremum norm $\|\cdot\|_{K}$. Then Bagchi's result can be formulated as follows.

The first author was partially supported by JSPS Grants 21740024. The second author was partially supported by grant no. N N201 605940 from National Science Centre.

(C) 2012 Australian Mathematical Publishing Association Inc. 0004-9727/2012 \$16.00 
THEOREM A. The Riemann hypothesis holds if and only if, for every compact set $K \subset D$ with connected complement and for every $\varepsilon>0$,

$$
\liminf _{T \rightarrow \infty} \frac{1}{T} \mu\left\{\tau \in[0, T]:\|\zeta(s+i \tau)-\zeta(s)\|_{K}<\varepsilon\right\}>0 .
$$

Analogously, as was shown in [11], the Riemann hypothesis is also equivalent to the analogue of (1.1) with $\zeta(s)$ replaced by the logarithm of the Riemann zeta function.

In 2010 Nakamura [8] showed the following property, which might be called selfapproximation of the Riemann zeta function.

Theorem B. For every algebraic irrational number $d \in \mathbb{R}$, every compact set $K \subset D$ with connected complement and every $\varepsilon>0$,

$$
\liminf _{T \rightarrow \infty} \frac{1}{T} \mu\left\{\tau \in[0, T]:\|\zeta(s+i d \tau)-\zeta(s+i \tau)\|_{K}<\varepsilon\right\}>0 .
$$

Pańkowski [12] extended the above result to all irrational numbers $d$, and Garunkštis [4] and Nakamura [9] independently investigated self-approximation of the Riemann zeta function for nonzero rational numbers. Unfortunately, the papers [4] and [9] contain a gap in the reasoning of the main theorem, so actually their methods work only for the logarithm of the Riemann zeta function. A detailed discussion on this matter was presented in [11], where Nakamura and Pańkowski partially filled this gap and prove self-approximation of $\zeta(s)$ for $d=a / b$ with $|a-b| \neq 1, \operatorname{gcd}(a, b)=1$.

Noteworthy is the fact that to prove self-approximation for every irrational number, Pańkowski proved the following joint universality theorem which implies selfapproximation.

Theorem C. Let $d \in \mathbb{R} \backslash \mathbb{Q}$ and $K \subset D$ be a compact set with connected complement. Assume that $f, g \in H(K)$. Then, for every $\varepsilon>0$,

$$
\liminf _{T \rightarrow \infty} \frac{1}{T} \mu\left\{\tau \in[0, T]:\|\zeta(s+i \tau)-f(s)\|_{K}<\varepsilon \text { and }\|\zeta(s+i d \tau)-g(s)\|_{K}<\varepsilon\right\}>0 .
$$

On the other hand, from Kaczorowski et al. [6] we get the following selfapproximation theorem for shifts of the Riemann zeta function.

Theorem D. Let $K \subset D$ be a compact set with connected complement. Assume that $f, g \in H(K)$ and $\lambda \in \mathbb{C}$ are such that $K_{\lambda}:=\{s+\lambda: s \in K\} \subset D$ and $K \cap K_{\lambda}=\emptyset$. Then, for every $\varepsilon>0$,

$$
\liminf _{T \rightarrow \infty} \frac{1}{T} \mu\left\{\tau \in[0, T]:\|\zeta(s+i \tau)-\zeta(s+\lambda+i \tau)\|_{K}<\varepsilon\right\}>0 .
$$

In this paper we shall investigate for which parameters $\lambda \in \mathbb{C}, d \in \mathbb{R}$ the inequality

$$
\forall_{\varepsilon>0} \liminf _{T \rightarrow \infty} \frac{1}{T} \mu\left\{\tau \in[0, T]:\|\zeta(s+\lambda+i d \tau)-\zeta(s+i \tau)\|_{K}<\varepsilon\right\}>0
$$

holds, assuming that $K$ is a compact set lying in the right half of the critical strip $D$ or in the half plane of absolute convergence $\mathcal{D}$. We also prove some results concerning joint universality and joint value approximation of functions $\zeta(s+\lambda+i d \tau)$ and $\zeta(s+i \tau)$. 


\section{The right open half of the critical strip}

We start with the generalisation of Theorem $\mathrm{C}$, which can be easily proved by a slight modification of its proof.

Theorem 2.1. Let $K_{1}, K_{2} \subset D$ be compact sets with connected complement and $d \in$ $\mathbb{R} \backslash \mathbb{Q}$. Assume that $f \in H\left(K_{1}\right)$ and $g \in H\left(K_{2}\right)$. Then, for every $\varepsilon>0$,

$$
\liminf _{T \rightarrow \infty} \frac{1}{T} \mu\left\{\tau \in[0, T]:\|\zeta(s+i \tau)-f(s)\|_{K_{1}}<\varepsilon \text { and }\|\zeta(s+i d \tau)-g(s)\|_{K_{2}}<\varepsilon\right\}>0 .
$$

Corollary 2.2. Let $K \subset D$ be a compact set with connected complement and $d \in$ $\mathbb{R} \backslash \mathbb{Q}$. Assume that $f, g \in H(K)$ and $\lambda \in \mathbb{C}$ satisfies $K_{\lambda}:=\{s+\lambda: s \in K\} \subset D$. Then, for every $\varepsilon>0$,

$\liminf _{T \rightarrow \infty} \frac{1}{T} \mu\left\{\tau \in[0, T]:\|\zeta(s+i \tau)-f(s)\|_{K}<\varepsilon\right.$ and $\left.\|\zeta(s+\lambda+i d \tau)-g(s)\|_{K}<\varepsilon\right\}>0$.

Thus the following self-approximation result is an immediate consequence of Corollary 2.2.

Corollary 2.3. Let $K \subset D$ be a compact set, $d \in \mathbb{R} \backslash \mathbb{Q}$ and $\lambda \in \mathbb{C}$ be such that $K_{\lambda} \subset D$. Then, for every $\varepsilon>0$,

$$
\liminf _{T \rightarrow \infty} \frac{1}{T} \mu\left\{\tau \in[0, T]:\|\zeta(s+i \tau)-\zeta(s+\lambda+i d \tau)\|_{K}<\varepsilon\right\}>0 .
$$

The following theorem shows that in fact inequality (2.1) holds also for rational $d \neq 0,1$, when $\lambda \in i \mathbb{R}$.

Theorem 2.4. Let $K \subset D$ be a compact set, $d \neq 0,1$ and $\lambda \in \mathbb{R}$. Then, for every $\varepsilon>0$,

$$
\liminf _{T \rightarrow \infty} \frac{1}{T} \mu\left\{\tau \in[0, T]:\|\log \zeta(s+i \tau)-\log \zeta(s+i \lambda+i d \tau)\|_{K}<\varepsilon\right\}>0 .
$$

Proof. By Corollary 2.3 it is sufficient to prove the theorem only for rational numbers $d=a / b$. In this case the proof follows closely the proof of Theorem 1 in [4], so we shall simply give a sketch.

Notice first that

$$
\left\|\tau \frac{\log p}{2 \pi b}-\frac{\lambda \log p}{2 \pi(b-a)}\right\|<\delta \quad \text { for } p \leq z
$$

implies the inequality

$$
\left\|\tau \frac{d \log p}{2 \pi}+\frac{\lambda \log p}{2 \pi}-\tau \frac{\log p}{2 \pi}\right\|<|a-b| \delta \quad \text { for } p \leq z,
$$

where $\|\cdot\|$ denotes the distance to the nearest integer.

Then by the continuity of a truncated zeta function $\zeta_{z}(s):=\prod_{p \leq z}\left(1-p^{-s}\right)^{-1}$ we have that for every $\varepsilon>0$ there exists $\delta>0$ such that

$$
\left\|\log \zeta_{z}(s+i \lambda+i d \tau)-\log \zeta_{z}(s+i \tau)\right\|_{K}<\varepsilon \quad \text { for all } \tau \text { satisfying (2.2) }
$$


Next, as in [4], one proves that

$$
\liminf _{T \rightarrow \infty} \frac{1}{T} \mu\left\{\begin{array}{ll} 
& \left\|\log \zeta(s+i \lambda+i d \tau)-\log \zeta_{z}(s+i \lambda+i d \tau)\right\|_{K}<\varepsilon \\
\tau \in[0, T]: & \left\|\log \zeta(s+i \tau)-\log \zeta_{z}(s+i \tau)\right\|_{K}<\varepsilon \\
\tau \text { satisfies }(2.2)
\end{array}\right\}>0
$$

for sufficiently large $z>0$. Modifications needed are easy and straightforward, so we skip them.

Finally, combining the last two formulas completes the proof.

\section{The half plane of absolute convergence}

Obviously, since the Riemann zeta function is absolutely convergent in the half plane $\mathcal{D}$, we cannot expect a universality theorem in this region. In spite of this fact, Bohr in [2] succeeded in proving that, in any strip $1<\sigma<1+\varepsilon, \zeta(s)$ takes any nonzero value infinitely often. Similarly, Mishou [7, Theorem 4] proved the following joint denseness result for the Riemann zeta function $\zeta(s)$ and the Hurwitz zeta function $\zeta(s ; \alpha)$.

Theorem E. Suppose that $\alpha$ is an algebraic irrational number with $0<\alpha<1$. Let $z_{1}$, $z_{2}$ be complex numbers. Then there exists a number $\sigma_{0}>1$ such that, for all positive $\varepsilon$,

$$
\liminf _{T \rightarrow \infty} \frac{1}{T} \mu\left\{\tau \in[0, T]:\left|\zeta\left(\sigma_{0}+i \tau\right)-z_{1}\right|<\varepsilon \text { and }\left|\zeta\left(\sigma_{0}+i \tau ; \alpha\right)-z_{2}\right|<\varepsilon\right\}>0 .
$$

Following the proof of Mishou's theorem, we prove the following result.

Theorem 3.1. Let $z_{1}, z_{2} \in \mathbb{C}, \varepsilon>0$ and $d \in \mathbb{R} \backslash \mathbb{Q}$. If we take $\delta>0$ sufficiently small, then for any $1<\sigma_{1}, \sigma_{2}<1+\delta$,

$$
\liminf _{T \rightarrow \infty} \frac{1}{T} \mu\left\{\tau \in[0, T]:\left|\zeta\left(\sigma_{1}+i \tau\right)-z_{1}\right|<\varepsilon \text { and }\left|\zeta\left(\sigma_{2}+i d \tau\right)-z_{2}\right|<\varepsilon\right\}>0 .
$$

In order to prove the above theorem, we require some notation and lemmas. The next lemma can be obtained by the method of the proof of [7, Lemma 4].

Lemma 3.2. Let $z \in \mathbb{C}$. If we take $\delta>0$ sufficiently small, then for any $\sigma_{0}$ with $1<\sigma_{0}<1+\delta$ there exist $\omega_{p}$ such that $\left|\omega_{p}\right|=1$ and

$$
\sum_{p} \log \left(1-\frac{\omega_{p}}{p^{\sigma_{0}}}\right)^{-1}=z
$$

The following lemma, proved by Pańkowski, plays an important role in the proof of Theorem 3.1 .

Lemma 3.3 (see [12, Lemma 2.4]). Let $\mathcal{P}$ be the set of all primes. For an arbitrary irrational number $d$, there exists a finite set of primes $A_{d}$ containing at most two elements such that the set $\{\log p\}_{\mathcal{P} \backslash A_{d}} \cup\left\{\log p^{d}\right\}_{\mathcal{P}}$ is linearly independent over $\mathbb{Q}$. 
Fix $d \in \mathbb{R} \backslash \mathbb{Q}$, and assume that $A:=A_{d}=\left\{a_{1}, a_{2}\right\}$ in the above lemma. Let

$$
\alpha_{h} \log a_{h}:=\sum_{n=1}^{m} \beta_{h, n} \log p_{n}+\sum_{n=1}^{m} \gamma_{h, n} \log p_{n}^{d},
$$

where $h=1,2$ and $\alpha_{h}, \beta_{h, n}, \gamma_{h, n}$ are suitable integers. By Lemma 3.3, the set

$$
\left\{\log p_{n}\right\}_{\mathcal{P} \backslash B} \cup\left\{\log p_{n}^{d}\right\}_{\mathcal{P}}, \quad B:=\left\{a_{1}, a_{2}, p_{1}, \ldots, p_{m}\right\},
$$

is linearly independent over $\mathbb{Q}$. Obviously, the set $\left\{\log p_{n}\right\}_{B \backslash A} \cup\left\{\log p_{n}^{d}\right\}_{B \backslash A}$ is linearly independent over $\mathbb{Q}$. Hence

$$
\left|a_{h}^{i \alpha_{h} \tau}-1\right|<\sum_{n=1}^{m}\left(\left|\beta_{h, n}\right|+\left|\gamma_{h, n}\right|\right) \varepsilon
$$

if $\tau$ satisfies the condition $\max _{p \in B \backslash A}\left|p^{i \tau}-1\right|<\varepsilon$ and $\max _{p \in B \backslash A}\left|p^{i d \tau}-1\right|<\varepsilon$. Put

$$
\zeta_{B}^{*}(s):=\zeta(s) \prod_{p \in B}\left(1-p^{-s}\right) .
$$

Proof of Theorem 3.1. By the proof of Lemma 3.2, we can omit a fixed number of primes and the statement of this lemma still holds. Therefore, let $z \in \mathbb{C}$. If we take $\delta>0$ sufficiently small, then for any $\sigma_{0}$ with $1<\sigma_{0}<1+\delta$ there exists $\omega_{p}$ such that $\left|\omega_{p}\right|=1$ and

$$
\sum_{2 \leq p<q} \log \left(1-\frac{1}{p^{\sigma_{0}}}\right)^{-1}+\sum_{p \geq q} \log \left(1-\frac{\omega_{p}}{p^{\sigma_{0}}}\right)^{-1}=z,
$$

where $q$ is some prime number and $p$ runs over all primes. By using the above equation and the fact that the set $\left\{\log p_{n}\right\}_{\mathcal{P} \backslash B} \cup\left\{\log p_{n}^{d}\right\}_{\mathcal{P}}$ is linearly independent over $\mathbb{Q}$, and modifying the proofs of [7, Theorem 4] and [8, Theorem 1.1],

$$
\liminf _{T \rightarrow \infty} \frac{1}{T} \mu\left\{\tau \in[0, T]: \begin{array}{c}
\left|\zeta_{B}^{*}\left(\sigma_{1}+i \tau\right) \prod_{p \in B}\left(1-p^{-\sigma_{1}}\right)^{-1}-z_{1}\right|<\varepsilon \\
\left|\zeta\left(\sigma_{2}+i d \tau\right)-z_{2}\right|<\varepsilon
\end{array}\right\}>0 .
$$

From the viewpoint of hybrid universality (see [5, Lemma 6] or [13, Theorem 4.1]) we can see that the above inequality is true if the condition $\max _{p \in B \backslash A}\left|p^{i \tau}-1\right|<\varepsilon$ is added. Thus we obtain Theorem 3.1.

Obviously, if we put $z_{1}=z_{2}$ in Theorem 3.1, then we obtain the following corollary. Corollary 3.4. Let $d \in \mathbb{R} \backslash \mathbb{Q}$ and $\varepsilon>0$. If we take $\delta>0$ sufficiently small, then for any $1<\sigma_{1}, \sigma_{2}<1+\delta$,

$$
\liminf _{T \rightarrow \infty} \frac{1}{T} \mu\left\{\tau \in[0, T]:\left|\zeta\left(\sigma_{1}+i \tau\right)-\zeta\left(\sigma_{2}+i d \tau\right)\right|<\varepsilon\right\}>0 .
$$


Next, we show that the self-approximation property holds for every compact set in the region of absolute convergence. In fact, we prove the following more general result for every function defined as an absolutely convergent general Dirichlet series.

Proposition 3.5. Let $d \in \mathbb{R}, F(s)=\sum_{n=1}^{\infty} a(n) e^{-\lambda_{n} s}\left(\lambda_{n} \in \mathbb{R}\right)$ be absolutely convergent for $\operatorname{Re} s>\sigma_{a}$ and $K$ be a compact set in the half plane $\sigma>\sigma_{0}$. Then, for every $\varepsilon>0$,

$$
\liminf _{T \rightarrow \infty} \frac{1}{T} \mu\left\{\tau \in[0, T]:\|F(s+i \tau)-F(s+i d \tau)\|_{K}<\varepsilon\right\}>0 .
$$

Proof. By absolute convergence, it suffices to show the assertion for $F_{N}(s)=$ $\sum_{n \leq N} a(n) e^{-\lambda_{n} s}$ with sufficiently large $N \in \mathbb{N}$.

Notice that for all $s \in K$,

$$
|F(s+i d \tau)-F(s+i \tau)| \leq \sum_{n \leq N} \frac{|a(n)|}{e^{\lambda_{n} \sigma}}\left|e^{\lambda_{n} i d \tau}-e^{\lambda i \tau}\right| \ll \max _{n \leq N}\left\|\lambda_{n} d \tau-\lambda_{n} \tau\right\| .
$$

Let $V$ denote the vector space over $\mathbb{Q}$ generated by the numbers $d \lambda_{n}$ and $\lambda_{n}$ for $n \leq N$. Assume that $\left\{\beta_{1}, \ldots, \beta_{k}\right\}$ is a basis of the space $V$. Then there exists a positive integer $M$ such that all numbers $d \lambda_{n}$ and $\lambda_{n}$ for $n \leq N$ can be represented as linear combinations of $\beta_{1} / M, \ldots, \beta_{k} / M$ with integer coefficients.

Hence, for every $\tau$ satisfying $\max _{1 \leq j \leq k}\left\|\tau \beta_{k} / M\right\|<\delta$,

$$
\max _{1 \leq n \leq N} \max \left(\left\|\tau d \lambda_{n}\right\|,\left\|\tau \lambda_{n}\right\|\right) \ll \delta .
$$

Thus, taking sufficiently small $\delta>0$ and using the classical Kronecker approximation theorem completes the proof.

Corollary 3.6. Let $K \subset \mathcal{D}$ be a compact set and $d \in \mathbb{R}$. Then, for every $\varepsilon>0$,

$$
\liminf _{T \rightarrow \infty} \frac{1}{T} \mu\left\{\tau \in[0, T]:\|\zeta(s+i d \tau)-\zeta(s+i \tau)\|_{K}<\varepsilon\right\}>0 .
$$

It turns out that (2.1) holds also in the half plane $\mathcal{D}$ for $\lambda \in i \mathbb{R}$.

Theorem 3.7. Let $1 \neq d \in \mathbb{R}$ and $\lambda \in \mathbb{R}$. Then, for every $\varepsilon>0$ and $K \subset \mathcal{D}$,

$$
\liminf _{T \rightarrow \infty} \frac{1}{T} \mu\left\{\tau \in[0, T]:\|\zeta(s+i \lambda+i d \tau)-\zeta(s+i \tau)\|_{K}<\varepsilon\right\}>0 .
$$

Proof. Let us observe that for every $z>0$,

$$
\left\|\zeta_{z}(s+i \lambda+i d \tau)-\zeta_{z}(s+i \tau)\right\|_{K}<\varepsilon
$$

whenever

$$
\max _{p \leq z}\left\|\tau \frac{d \log p}{2 \pi}+\frac{\lambda \log p}{2 \pi}-\tau \frac{\log p}{2 \pi}\right\|<\delta .
$$

By unique factorisation of integers, we see that the numbers $(d-1) \log p / 2 \pi(p \leq z)$ are linearly independent over $\mathbb{Q}$ for every real $d \neq 1$. Then, by the Kronecker theorem and absolute convergence of the Riemann zeta functions in $\mathcal{D}$, the proof is complete. 
At the end of this section we deal with the case where $\lambda \in \mathbb{C}$ with $\operatorname{Re} \lambda>0$. We show that in this case we cannot expect the self-approximation property to hold. In order to prove thus we need the following lemma.

Lemma 3.8. For every $\lambda \in \mathbb{C}$ with $\operatorname{Re} \lambda>0$ there is $\sigma_{0}>1$ such that for all $s$ with $\operatorname{Re} s>\sigma_{0}$, all $t, d \in \mathbb{R}$,

$$
|\zeta(s+\lambda+i t)-\zeta(s+i d t)|>c:=c(\lambda)>0 .
$$

Proof. Let us observe that for all $s$ with $\operatorname{Re} s:=\sigma>1$,

$$
|\zeta(s+\lambda)-1| \leq \sum_{n \geq 2} \frac{1}{n^{\sigma+\operatorname{Re} \lambda}}, \quad|\zeta(s)-1| \geq 2^{-\sigma}-\sum_{n \geq 3} n^{-\sigma} .
$$

Then, since $d$ and $t$ are real numbers, it suffices to prove that for a given $\lambda \in \mathbb{C}$ with $\operatorname{Re} \lambda>0$ there exists $\sigma_{0} \geq 2$ such that, for every $\sigma>\sigma_{0}$,

$$
\sum_{n \geq 2} \frac{1}{n^{\sigma+\operatorname{Re} \lambda}}<2^{-\sigma}-\sum_{n \geq 3} n^{-\sigma}
$$

or, equivalently, that

$$
\sum_{n \geq 3} \frac{1}{n^{\sigma+\lambda}}+\sum_{n \geq 3} n^{-\sigma}<2^{-\sigma}\left(1-2^{-\operatorname{Re} \lambda}\right)
$$

Using the inequality

$$
\sum_{n \geq 3} n^{-\sigma} \leq 4 \cdot 3^{-\sigma} \text { for } \sigma \geq 2
$$

we obtain that (3.1) holds, provided

$$
4 \cdot 3^{-\sigma}\left(1+3^{-\operatorname{Re} \lambda}\right)<2^{-\sigma}\left(1-2^{-\operatorname{Re} \lambda}\right) .
$$

Thus the proof is complete, since $\operatorname{Re} \lambda>0$ and $(2 / 3)^{\sigma} \rightarrow 0$ when $\sigma \rightarrow \infty$.

Theorem 3.9. For every $\lambda \in \mathbb{C}$ with $\operatorname{Re} \lambda>0$ there exist $K \subset \mathcal{D}$ and $c>0$ such that, for all real $t$ and $d$,

$$
\|\zeta(s+\lambda+i t)-\zeta(s+i d t)\|_{K}>c .
$$

Proof. Let $\sigma_{0}$ be such that the inequality from the previous lemma holds. Then the proof is complete for any set $K$ satisfying $K \cap\left\{s: \operatorname{Re} s>\sigma_{0}\right\} \neq \emptyset$.

Let us observe that, for $\lambda \in \mathbb{C}$ with small real part, the set $K$ chosen in the above proof is rather wide, namely the value $\max _{s \in K} \operatorname{Re} s-\min _{s \in K} \operatorname{Re} s$ is large. Nevertheless, if we assume that $d=1$ and $\mathbb{R} \ni \lambda>0$, then one can prove that the set $K$ can also be very narrow, even for small $\lambda$.

Proposition 3.10. For every $\mathbb{R} \ni \lambda>0$ there exist $K \subset \mathcal{D}$ and $c>0$ such that, for all real $\tau$,

$$
\|\zeta(s+\lambda+i \tau)-\zeta(s+i \tau)\|_{K}>c .
$$


To prove the above proposition, we recall that a function $f(s)$, defined on some vertical strip $a<\sigma<b$, is called almost periodic if, for any $\varepsilon>0$ and any $\alpha, \beta$ with $a<\alpha<\beta<b$, there exists a length $l=l(f, \alpha, \beta, \varepsilon)>0$ such that for every interval $\left(t_{1}, t_{2}\right)$ of length $l$ there exists $\tau \in\left(t_{1}, t_{2}\right)$ satisfying

$$
|f(\sigma+i t+i \tau)-f(\sigma+i t)|<\varepsilon \quad \text { for every } \alpha<\sigma<\beta, t \in \mathbb{R} .
$$

We use the fact, proved by Bohr, that every Dirichlet series is almost periodic in the half plane $\sigma>\sigma_{a}$ of absolute convergence.

Proof. Let us take an arbitrary $\lambda>0$ and $s=\sigma+i t$ with $\sigma>1$. We put $l=l(\zeta, \sigma-\delta$, $\sigma+\lambda+\delta, \varepsilon$ ), where $0<\delta<\sigma-1$ and $\varepsilon>0$ will be fixed later. Next let us define $K=[\sigma-\delta / 2, \sigma+\delta / 2] \times[0, l]$.

Thus, for every $\tau \in \mathbb{R}$ there exists $\tau^{\prime} \in[\tau, \tau+l]$ such that

$$
\left|\zeta\left(\sigma+i \tau^{\prime}\right)-\zeta(\sigma)\right|<\varepsilon \text { and }\left|\zeta\left(\sigma+\lambda+i \tau^{\prime}\right)-\zeta(\sigma+\lambda)\right|<\varepsilon .
$$

Hence

$$
\left|\zeta\left(\sigma+i \tau^{\prime}\right)-\zeta\left(\sigma+\lambda+i \tau^{\prime}\right)\right| \geq|\zeta(\sigma+\lambda)-\zeta(\sigma)|-2 \varepsilon .
$$

Using the fact that $\zeta(\sigma)$ is a strictly decreasing function for $\mathbb{R} \ni \sigma>1$ and taking sufficiently small $\varepsilon>0$ and $\delta>0$ completes the proof.

\section{The half plane $\operatorname{Re} s>1 / 2$}

In this final section we investigate the case where $\lambda \in \mathbb{C}$ in (2.1) is such that $K_{\lambda} \subset \mathcal{D}$ for a given compact set $K$ lying in the right half of the critical strip.

We start with the following combination of universality and value approximation property.

THEOREM 4.1. Let $z \in \mathbb{C}$ and $f(s) \in H(K)$, where $K \subset D$ is a compact set with connected complement. Then for any $\varepsilon>0, d \in \mathbb{R} \backslash \mathbb{Q}$, there exists $\sigma_{0}>1$ such that

$$
\liminf _{T \rightarrow \infty} \frac{1}{T} \mu\left\{\tau \in[0, T]:\|\zeta(s+i \tau)-f(s)\|_{K}<\varepsilon \text { and }\left|\zeta\left(\sigma_{0}+i d \tau\right)-z\right|<\varepsilon\right\}>0 .
$$

Proof. Using the fact that $\left\{\log p_{n}\right\}_{\mathcal{P} \backslash B} \cup\left\{\log p_{n}^{d}\right\}_{\mathcal{P}}$ is linearly independent over $\mathbb{Q}$ and modifying the proofs of [7, Theorem 4] and [8, Theorem 1.1], or [12, Theorem 1.1], there exists $\sigma_{0}>1$ such that

$$
\liminf _{T \rightarrow \infty} \frac{1}{T} \mu\left\{\tau \in[0, T]:\left\|\zeta_{B}(s+i \tau)-f(s)\right\|_{K}<\varepsilon \text { and }\left|\zeta\left(\sigma_{0}+i d \tau\right)-z\right|<\varepsilon\right\}>0 .
$$

By hybrid universality (see, for example, [13]), we can see that the above inequality also holds if the condition $\max _{p \in B \backslash A}\left|p^{i \tau}-1\right|<\varepsilon$ is added. Therefore

$$
\liminf _{T \rightarrow \infty} \frac{1}{T} \mu\left\{\tau \in[0, T]: \begin{array}{c}
\left\|\zeta(s+i \tau)-f(s) \prod_{p \in B}\left(1-p^{-s}\right)\right\|_{K}<\varepsilon \\
\left|\zeta\left(\sigma_{0}+i d \tau\right)-z_{2}\right|<\varepsilon
\end{array}\right\}>0 .
$$

Hence we obtain Theorem 4.1. 
THeOREM 4.2. Assume that, for a given compact set $K \subset D$ and $\lambda \in \mathbb{C}$, the set $K_{\lambda}:=$ $\{s+\lambda: s \in K\} \subset \mathcal{D}$. Then for any $\varepsilon>0, d \in \mathbb{R}$,

$$
\liminf _{T \rightarrow \infty} \frac{1}{T} \mu\left\{\tau \in[0, T]:\|\zeta(s+i \tau)-\zeta(s+\lambda+i d \tau)\|_{K}<\varepsilon\right\}>0 .
$$

Proof. Note that $\zeta(s+\lambda)$ is nonvanishing and convergent absolutely in $s \in K$ by the assumption $K_{\lambda} \subset \mathcal{D}$. By modifying the proof of Theorem 4.1 and [10, Theorem 1],

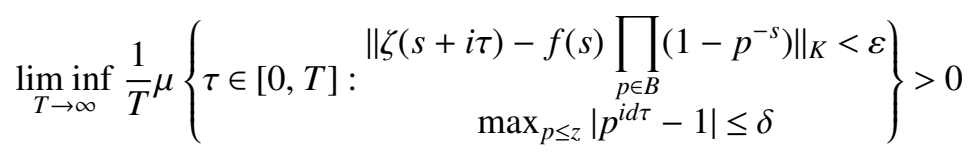

for every $\delta>0$ and $z>0$. Then putting $f(s) \prod_{p \in B}\left(1-p^{-s}\right)=\zeta(s+\lambda)$ and using the reasoning of Proposition 3.5 for sufficiently small $\delta$ and sufficiently large $z$, we complete the proof.

\section{References}

[1] B. Bagchi, 'Recurrence in topological dynamics and the Riemann hypothesis', Acta Math. Hung. 50 (1987), 227-240.

[2] H. Bohr, 'Über das Verhalten von $\zeta(s)$ in der Halbebene $\sigma>1$ ', Nachr. Akad. Wiss. Gottingen Math.-Phys. Kl. II (1911), 409-428.

[3] H. Bohr, 'Über eine quasi-periodische Eigenschaft Dirichletscher Reihen mit Anwendung auf die Diriehletschen L-Funktionen', Math. Ann. 85(1) (1922), 115-122.

[4] R. Garunkštis, 'Self-approximation of Dirichlet $L$-functions', J. Number Theory 131(7) (2011), 1286-1295.

[5] J. Kaczorowski and M. Kulas, 'On the non-trivial zeros off line for $L$-functions from extended Selberg class', Monatsh. Math. 150 (2007), 217-232.

[6] J. Kaczorowski, A. Laurinčikas and J. Steuding, 'On the value distribution of shifts of universal Dirichlet series', Monatsh. Math. 147 (2006), 309-317.

[7] H. Mishou, 'The joint value distribution of the Riemann zeta function and Hurwitz zeta functions. II.', Arch. Math. (Basel) 90(3) (2008), 230-238.

[8] T. Nakamura, 'The joint universality and the generalized strong recurrence for Dirichlet $L$ functions', Acta Arith. 138(4) (2009), 357-362.

[9] T. Nakamura, 'The generalized strong recurrence for nonzero rational parameters', Arch. Math. 95 (2010), 549-555.

[10] T. Nakamura and $€$. Pańkowski, 'On universality for linear combinations of $L$-functions', Monatsh. Math. 165 (2012), 433-446.

[11] T. Nakamura and Ł. Pańkowski, 'Erratum to "The generalized strong recurrence for non-zero rational parameters", Arch. Math. (Basel) 99(1) (2012), 43-47.

[12] Ł. Pańkowski, 'Some remarks on the generalized strong recurrence for L-functions', in: New Directions in Value-Distribution Theory of Zeta and L-functions, Berichte aus der Mathematik (Shaker Verlag, Aachen, 2009), pp. 305-315.

[13] Ł. Pańkowski, 'Hybrid joint universality theorem for the Dirichlet L-functions', Acta Arith. 141(1) (2010), 59-72.

[14] J. Steuding, Value-Distribution of L-functions, Lecture Notes in Mathematics, 1877 (Springer, Berlin, 2007). 


\section{TAKASHI NAKAMURA,}

Department of Mathematics, Faculty of Science and Technology, Tokyo University of Science Noda, Chiba 278-8510, Japan

e-mail: nakamura_takashi@ma.noda.tus.ac.jp

ŁUKASZ PAŃKOWSKI, Faculty of Mathematics and Computer Science, Adam Mickiewicz University, Umultowska 87, 61-614 Poznań, Poland e-mail: lpan@amu.edu.pl 